\title{
ON THE ZEROES OF CERTAIN CLASSES OF INTEGRAL TAYLOR SERIES. PART I.-ON THE INTEGRAL FUNCTION
}

$$
\sum_{n=0}^{\infty} \frac{x^{\phi(n)}}{\{\phi(n)\} !} \text {. }
$$

\section{By G. H. HaRdY.}

[Received Augurt 20th, 1904.-Revised October, 1904.-Read November 10th, 1904,]

1. This paper and another which I hope to publish before long are intended as a contribution to the solution of the following problem:- "To find particular (but as large as possible) classes of integral Taylor series, such that the nature of the zeroes associated with the essential singularity at infinity can be determined with precision."

This problem is somewhat different from and less ambitious than the classical problem of the theory of integral functions, the object of the works of Poincaré, Hadamard, and their successors in this field. The degree of precision attained by the results proved in these papers is, as will be seen clearly later on, wholly incompatible with initial hypotheses of the same degree of generality as those adopted by the writers referred to. If we start, as they do, by supposing known merely certain limits for the increase (croissance) of the moduli of the reciprocals of the coefficients, all that is generally possible is to determine corresponding limits for the increase of the roots. This problem has, of course, up to a certain point, heen studied with conspicuous success; and it seems as if all that can be done further in this direction is to impart some additional precision to the known results.*

- The original memoirs of Poincaré and Hadamard were: Poincaré, "Sur les fonctions entières" (Bull. de la Soc. Math. de France, 1883); Hadamard, “Etude sur les propriétés des fonctions entières" (Jourmal de Math., 1893, p. 171).

Numerous references to further writings on the subject up to 1900 will be found in M. Borel's Lecons sur les fonctions :ntières, 1900.

Among later publications I may mention: P. Boutroux, "Sur quelques propriétés des fonctions entières" (Acta Mrath., Vol. xxvir., p. 97); E. Lindelöf, "Mémoire sur les fonctions entières de genre fini" (Acta Soc. Fennicae, Vol. xxxI., p. 1) ; E. W. Barnes, "A Memoir on Integral Functions" (Phil. Truth., A, Vol. 199, p. 411); A. Wiman, "Sur le cas d'exception dans la théorie des fonctions entières" (Arkiv for Mat. Ast. och Fysik, Vol. 1., p. 327); Edm. Maillet, "Sur les fonctious entières et quasi-entières" (Journal de Math., 1902, p. 329); J.-L.-W.-V. Jensen, "Sur un nouvel et important théorème de la théorie des fonctions," Acta Math., Vol. xxir.

There are other writings, too numerous to mention, by MM. Lindelöf, Wiman, and others. 
My object in these and in some earlier papers* on particular integral functions has been to "find asymptotically" the zeroes of the function under consideration; that is to say, to determine an enumerable sequence of points such that, if $\sigma$ be an arbitrarily small positive quantity, and circles of radius $\sigma$ be described with the points as centres, we can choose $R$ so that all the zeroes whose distance from the origin is greater than $R$ lie within the circles, each circle containing one and only one.t

It is evident that to have any hope of obtaining results as precise as this we must confine ourselves to "particular cases." In the case of a Taylor series, for instance, we must in general suppose known not merely the increase of the reciprocals of the coefficients, but also their analytical nature. At the same time what is, from the point of view of the general theory, an altogether special case may still include a considerable variety of interesting functions; and, for this reason, I hope that the analysis contained in this and the following paper may be of some interest to those who are engaged in the study of the general theory of integral functions.

The first step in all such investigations must necessarily be the determination of asymptotic representations of the function some one of which is valid in every region of the plane. When this has been lone the asymptotic determination of the zeroes is generally easy enough; at any rate, it is generally easy enough to find a series of points in whose neighbourhood the zeroes must be sought for. To find a precise proof that one and only one zero is associated with each point is sometimes a matter of greater difficulty.

It will be generally found, with functions such as we are naturally led to consider, that the whole plane with the exception of certain "barrier: regions " $E$ can be divided into a number of regions $D$, within which the function is given asymptotically by an equation of the form

$$
f(x)=\phi_{D}(x)\left(1+\rho_{x}\right),
$$

$\rho_{x}$ being a function of $x$ which tends uniformly to zero with $1 / x$, and $\phi_{D}(x)$ a function which has no zeroes in $D$. It then follows that $f(x)$ has no

" "On the Zeroes of the Integral Function $x-\sin x "$ (Messenger, Vol. xxxı., p. 161) ; "On the Zeroes of certain Integral Functions" (Messenger, Vol. xxxu., p. 36) ; "The Asymptotic Solution of certain Transcendental Equations" (Quarterly Journal, Vol. xxxv., p. 261); "On the Roots of the Equation $\frac{1}{\Gamma(x+1)}=c "$ (Proc. London Math. Soc., Ser. 2, Vol. 2, p. 1); “ Note on an Integral Function" (Messenger, Vol. xxxIv., p. 1).

+ The approximation is not always quite as precise as this. 
zeroes except perhaps in the barrier regions $E$. Suppose, for instance, that there are two regions $D$ separated by a single region $E$. Then the asymptotic determination of the zeroes will be found to rest upon a proof that within the region $E$

$$
f(x)=\phi_{D}(x)\left(1+\rho_{x}\right)+\phi_{D^{\prime}}(x)\left(1+\rho_{x}\right),
$$

and that the zeroes are to be found near the points for which

at any rate asymptotically.

$$
0=\phi_{D}(x)+\phi_{D^{\prime}}(x),
$$

In these two papers I deal with two classes of functions closely connected with the ordinary exponential function. I have no doubt that similar investigations might be carried out for classes of functions similarly formed from other standard functions-from Prof. MittagLeffler's function

$$
E_{a}(x)=\sum_{0}^{\infty} \frac{x^{n}}{\Gamma(a n+1)}, *
$$

for example. I have not attempted to carry out such investigations for the reason that Prof. Mittag-Leffler's long promised memoir on the function $E_{a}(x)$ has not yet appeared. $\dagger$

Of the two classes of functions which I consider the second $\ddagger$ seems to me the more interesting in itself. Theoretically, however, the first is perhaps more so, on account of the extreme simplicity of the method employed, which proceeds directly from the Taylor series, depending merely on in development of the idea, originally due to M. Borel, of the preponderant importance for certain functions of the numerically greatest term in the expansion. In dealing with the second class a preliminary transformation of the series into the more easily manipulated form of a definite integral seems to be essential.\$

2. The first class of functions is that formed by a suitable selection of terms from the exponential series itself, that is to say the class of functions defined by a series of the form

$$
\sum_{n=0}^{\infty} \frac{x^{\phi(n)}}{\{\phi(n)\} !}
$$

- Mittag-Leffler, Comptes Rendtss (2 Mars, 1903).

+ I do not know whether Prof. Mittag-Leffler has asymptotically determined the zeroes of $E_{\mathrm{a}}(x)$.

† The functions $\Sigma \frac{x^{n}}{\Gamma a+n+1)}, \Sigma \frac{x^{n}}{(n+a)^{n} n !}$ are typical members of this class.

$\$$ A third class of functions whose zeroes may be investigated by very elementary methods is the class defined by series of the type $\Sigma \frac{x^{n}}{b_{0} b_{1} \ldots b_{n}}$, when the increase of $\left|b_{n}\right|$ is rapid. 
where $\phi(n)$ is a function of $n$ which is positive and integral for all positive integral values of $n$, and increases steadily with $n$. It is very easy to see that the increase of such a function cannot be very far removed from that of $e^{x}$. In the first place, if the maximum of the modulus of the function is denoted by $M(r)$, it is obvious that

$$
M(r) \leqslant e^{r}
$$

But, on the other hand, we obtain an inferior limit for $M(r)$ by considering the increase of the greatest term in the expansion of $e^{r}$. Now this term is the one for which

$$
r / n \geqslant 1>r /(n+1)
$$

i.e., the $[r]$-th. The value of this is

$$
r^{r} / r ! \sim e^{r} / \sqrt{ }(2 \pi r){ }^{*}
$$

Hence it follows that the increase of all the functions with which we are concerned lies between that of $e^{r}$ and that of $e^{r} / \sqrt{ } r$. In M. Borel's notationt we may say that, if $\iota$ is the order of infinity of $M(r)$ for $r=\infty$, then

$$
\omega-\frac{1}{2} \leqslant \iota \leqslant \omega .
$$

It is, of course, only for certain forms of the function $\phi$, which we may call normal forms, that the precise determination which is desired can be made. Moreover, for it to be practicable it is essential (in general) that the increase of $\phi(n)$ should be sufficiently rapid. I shall first work out in detail what appears to be the simplest case, that in which

$$
\begin{gathered}
\phi(n)=n^{3}, \\
f(x)=\sum_{n=0}^{\infty} \frac{x^{n^{3}}}{n^{3 !}},
\end{gathered}
$$

and then indicate the various generalisations which can be made.

$$
\text { The Function } f(x)=\sum_{n=0}^{\infty} \frac{x^{n^{3}}}{n^{8} !} .
$$

3. It follows from Stirling's theorem that

$$
n^{3} !=n^{3 n^{3}+\frac{7}{2}} e^{-n^{3}} \sqrt{ }(2 \pi)(1+\rho),
$$

\footnotetext{
- By $f(a) \sim \phi(a)$ I mean, in this paper, $\lim _{a=-\infty} f / \phi=1$; the symbol was introduced by Du Bois-Reymond.

† See his Leçons sur les séries à termes positifs, chap. iii.
} 
where

$$
|\rho|<K / n^{3} .^{*}
$$

Suppose that

$$
x=r e^{i \theta}, \quad v_{n}=\left|x^{n^{3}}\right| n^{3} ! \mid \text {. }
$$

Then we find by an easy calculation that

$$
v_{n} / v_{n+1}=\left(n^{3} / r\right)^{3 n^{3}+3 n+1} e^{f(n+1)}(1+\rho) \quad(|\rho|<K / n) .
$$

If $r=N^{3}$, where $N$ is a large integer,

$$
v_{N} / v_{N+1}=e^{f(N+1)}(1+\rho) \quad(|\rho|<K / N)
$$

while, if $n>N$,

$$
v_{n} / v_{n+1}>v_{N} / v_{N+1}>e^{q\left(N^{N}+1\right)}(1+\rho) .
$$

We can fix a large integer $N_{0}$, and then take $N$ large in comparison with $N_{0}$. Then, if $N_{0} \leqslant n<N, r=N^{3} \geqslant(n+1)^{3}$, and

$$
\frac{v_{n}}{v_{n+1}}<K\left(\frac{n}{n+1}\right)^{9 n^{2}+9 n+3} e^{\frac{8}{(2 n+1)}}<K e^{-\frac{8 n}{2 n}} .
$$

From (3), $\left(3^{\prime}\right)$, and $\left(3^{\prime \prime}\right)$ it follows that

$$
\sum_{N_{10}}^{\infty} \frac{x^{n^{3}}}{n^{3} !}=\frac{x^{N^{3}}}{N^{3} !}(1+\rho) \quad(|\rho|<K / N) .
$$

And thus it is evident that, if $N$ is sufficiently large in comparison with $N_{0}$,

$$
f(x)=\sum_{0}^{\infty} \frac{x^{n^{3}}}{n^{3} !}=\frac{x^{N^{3}}}{N^{3} !}(1+\rho),
$$

when $r=N^{3}$. Now $\frac{x^{N^{3}}}{N^{3} !}=\frac{N^{3 N^{3}}}{N^{3} !} \exp \left(N^{3} i \theta\right)$,

$$
\frac{N^{3 N^{3}}}{N^{3} !}=\frac{e^{N^{3}}}{\sqrt{ }\left(2 \pi N^{3}\right)}(1+\rho)=\frac{e^{r}}{\sqrt{ }(2 \pi r)}(1+\rho) .
$$

Thus along the circle of radius $r=N^{3}$

$$
f(x)=(2 \pi r)^{-\frac{\hbar}{2}} e^{r+r i \theta}(1+\rho),
$$

where $|\rho|<K / r^{3}$. We have thus defined a series of circles round each of which the modulus of $f(x)$ is large, being substantially of the order of $e^{r} / \sqrt{ } r$.

4. From this formula it is evident that when $x$ travels round the circle of radius $N^{9}$ the amplitude of $f(x)$ is increased by $2 \pi N^{3}$. Hence it follows

- In this paper I use $K$ to denote a number not the same in different ineyualities, but always lying betwreen certain fixed limits, say $1,000,000$ and $1 / 1,000,000$. I need hardly say that $\rho$ also is not the same in different inequalities or equations. 
that, if $N$ is sufficiently large, there are exactly $N^{3}$ zeroes of $f(x)$ within a circle whose centre is the origin and whose radius is $N^{3}$.

I shall now proceed to determine more precisely the positions of the $3 N^{2}+3 N+1$ zeroes which lie between the circles $r=N^{3}, r=(N+1)^{3}$.

5. Suppose then that $N^{3}<r<(N+1)^{3}$.

If $n \geqslant N+1, \quad \quad v_{n} / v_{n+1}>e^{\nexists n}$,

while, if $N_{0} \leqslant n \leqslant N-1, \quad v_{n} / v_{n+1}<K e^{-\frac{3}{3} n}$.

Hence it follows that

$$
f(x)=\frac{x^{N^{3}}}{N^{3 !}}(1+\rho)+\frac{x^{(N+1)^{3}}}{(N+1)^{3} !}(1+\rho) \quad(|\rho|<K / N) .
$$

As $r$ increases from $N^{3}$ to $(N+1)^{3}$ the importance of the second term grows at the expense of the first, and, if $x$ is situated at one of the zeroes, it is evident that

$$
x^{3 N^{3}+3 N+1}=-\frac{(N+1)^{3} !}{N^{3} !}(1+\rho)
$$

or

$$
r^{3 N^{y}+3 N+1}=\frac{(N+1)^{3} !}{N^{3} !}(1+\rho)
$$

and

$$
e^{\left(3 N^{3}+3 N+1\right) i \theta}=e^{(2 k+1) i \pi}(1+\rho),
$$

$k$ being an integer. From these two equations we easily deduce

$$
r=N^{3}(1+3 / 2 N+\ldots)
$$

and

$$
\theta=\frac{2 k+1}{3 N^{2}+3 N+1} \pi+\rho \quad\left(k=0,1, \ldots, 3 N^{2}+3 N\right) .
$$

It would be easy to carry the approximation further, but the above formulæ give as much information as is necessiry for my present purpose. I may remark in passing that we can alwiys find a lower as well as an upper limit for the number of zeroes of a function $f(x)$ lying within a circle of radius $r$, if $|f(x)|$ is large for all points on the circle. These limits may be at once deduced from M. Jensen's well known formula expressing $\int_{0}^{2 \pi} \log |f(x)| d \theta$ in terms of the zeroes.

SKR. 2. VOL. 2. NO. 872 . 
Thus the $3 N^{2}+3 N+1$ zeroes of $f(x)$ which lie between the circles $r=N^{3}$ and $r=(N+1)^{3}$ are given approximately by some or all of the $3 N^{2}+3 N+1$ points defined by the equations (10) and (11).

It is natural to suppose that one and only one root of $f(x)$ is thus associated with each of these points; and this is, in fact, the case. I have not thought it worth while to set out the proof of this in detail, as I have given formal proofs of similar points with respect to other functions on several occasions, ${ }^{*}$ and the present case is not one in which any point of particular interest or difficulty arises.

The function $f(x)$ is therefore (roughly speaking) what Mr. Barnest has called a ring function; its zeroes lie (roughly speaking) on circles, separated by circles along which its modulus is everywhere large.

It is easy to see that, if we exclude the zeroes of $f(x)$ from the plane by a series of circles with their centres at the zeroes and of fixed, but arbitrarily small, radius, then throughout the rest of the plane

$$
|f(x)|>K r^{-\frac{1}{2}} e^{r} .
$$

The function has therefore the property that its modulus tends (in general) to infinity when $x$ approaches infinity in any direction.

\section{Generalisations.}

6. The preceding analysis at once suggests various generalisations. For it clearly depends only on the fact that a series of circles can be defined on which the behaviour of $f(x)$ is completely dominated by the behaviour of one of its terms, while between any two circles it is completely dominated by the behaviour of $t w o$. The analysis would therefore be equally practicable for any function of the form

$$
f(x)=\sum_{n=0}^{\infty} \frac{x^{\phi(n)}}{\{\phi(n)\} !},
$$

provided the increase of $\phi(n)$ is regular and sufficiently rapid; for instance, if $\phi(n)$ were

$$
n^{4}, n^{5}, \ldots,\left[n^{p} \log n\right](p \geqslant 3),\left[e^{n}\right], \ldots
$$

In general we find that the value of $f(x)$ on the circle $r=\phi(n)$ is given by the formula (5), just as in the special case when $\phi(n)=n^{3}$, so that the number of zeroes within the circle is exactly $\phi(n)$. Moreover, the form

- See the papers referred to above (p. 333),

+ L.c., p. 424 , 
of $f(x)$ may be altered in another way, as the argument applies equally well to

$$
F(x)=\sum_{n=0}^{\infty} \frac{\psi(n) x^{\phi(n)}}{\{\phi(n)\} !}
$$

provided that $\psi(n)$ satisfies certain conditions easily defined. In this case the form of (5) is of course slightly modified. Or, again, we might consider the function

$$
F_{1}(x)=\sum_{n=0}^{\infty} \frac{\psi(n) x^{\phi(n)}}{\{\phi(n)\}^{\phi(n)}}
$$

but the principle will have been sufficiently illustrated by what precedes. I may, however, remark that the argument will not apply in its present form to

$$
f(x)=\sum_{n=0} \frac{x^{n^{2}}}{n^{2} !},
$$

the increase of $n^{2}$ not being sufficiently rapid, as is easily seen on working out the necessary approximations.

[As a matter of fact asymptotic formulæ for $f(x)$ and for its zeroes, analogous to those found in the case when $\phi(n)=n^{3}$, may be determined; but the investigation is more difficult, and involves the theory of elliptic functions, and it is no longer true that the behaviour of $f(x)$ is dominated by that of one or two terms. I find that, for $r=N^{2}$,

$$
f(x)=\frac{e^{r+r i \theta}}{\sqrt{ }(2 \pi r)} \phi(\theta)(1+\rho),
$$

where

$$
\phi(\theta)=\sum_{-\infty}^{\infty} e^{\nu \mathbf{s}(i \theta-2)+2 \nu N i \theta} .
$$

In particular, if $\theta=0, \quad f(x)=\frac{A e^{x}}{\sqrt{ }(2 \pi x)}$,

where

$$
A=1+2 \sum_{1}^{\infty} e^{-2 \omega^{s}} \text {. }
$$

There are $N^{2}$ zeroes of $f(x)$ within a large circle of radius $N^{2}$, and the $2 N+1$ which lie between the circles $r=N^{2}, r=(N+1)^{2}$ are given approximately by

$$
r=N^{2}+N, \quad \theta=\frac{2 q-1}{2 N+1} \pi \quad(q=1,2, \ldots, 2 N+1) .
$$

-Added November 4th, 1904.] 